\title{
Acute cor pulmonale due to pulmonary tumor thrombotic microangiopathy in two patients with breast cancer
}

\author{
Sung Young Moon, Kang Hoon Lee, Jong Sik Lee, Hyun Suk Yang, Hong Ghi Lee, Yo Han Cho, \\ and So Young Yoon
}

Department of Internal Medicine, Konkuk University School of Medicine, Seoul, Korea

Received: April 27, 2015

Revised : July 3, 2015

Accepted: July 6, 2015

\section{Correspondence to}

So Young Yoon, M.D.

Department of Internal Medicine, Konkuk University School of Medicine, 120-1 Neungdong-ro, Gwangjin-gu, Seoul 05030, Korea

Tel: +82-2-2030-7537

Fax: +82-2-2030-7748

E-mail: greenteamd@gmail.com
To the Editor, Pulmonary tumor thrombotic microangiopathy (PTTM), a rare manifestation of cancer, was first reported by von Herbay et al. [1] in 1990; it is a fatal clinical syndrome characterized by rapid progression of dyspnea because of acute pulmonary hypertension, causing right heart failure and death within a few days of onset. The pathogenesis involves microscopic tumor-cell infiltration into small pulmonary vessels, inducing local paraneoplastic thromboses and fibrocellular intimal hyperplasia, resulting in stenosis and occlusion. Antemortem PTTM diagnosis is difficult because of rapid progression from onset of dyspnea to death; therefore, antemortem PTTM diagnosis in breast cancer is rare. We report two cases of PTTM in patients with breast cancer and discuss the pathogenesis and clinical course of PTTM.

A 45-year-old woman was diagnosed with triple negative, invasive ductal carcinoma within her left breast. She underwent left modified radical mastectomy, six cycles of adjuvant chemotherapy (docetaxel $75 \mathrm{mg} / \mathrm{m}^{2}$, doxorubicin $50 \mathrm{mg} / \mathrm{m}^{2}$, and cyclophosphamide $500 \mathrm{mg} / \mathrm{m}^{2}$ ), and postoperative radiation therapy (50.4 Gy). This patient's initial disease was stage IIIc with $\mathrm{T}_{3} \mathrm{~N}_{3} \mathrm{Mo}$. After 7 months, she had breast cancer relapse with left anterior chest wall, left supraclavicular, mediastinal, subcarinal lymph node, and multiple bone metastases. She received palliative chemotherapy consisting of gemcitabine 100 $\mathrm{mg} / \mathrm{m}^{2}$ on days 1 and 8 , and cisplatin 60 $\mathrm{mg} / \mathrm{m}^{2}$ on day 1 , repeated every 3 weeks. However, she was lost to follow-up after completing one cycle, and 6 months later she presented to the emergency department with a 3-week history of progressive dyspnea on exertion (New York Heart Association function class II). She had used complimentary medicine before admission and since the loss to follow-up. A chest radiograph showed clear lung field. There was no crackle or wheezing on chest auscultation. Arterial blood gas analysis results in room air indicated hypoxemia: $\mathrm{PaO}_{2}$, $47.4 \mathrm{mmHg}$; $\mathrm{SaO} 2,82.1 \%$. Blood analysis showed D-dimer elevated to $4.46 \mu \mathrm{g} / \mathrm{mL}$ (normal, o to o.4), with troponin I elevated to $0.2 \mathrm{ng} / \mathrm{mL}$ (normal, o to 0.02 ), brain natriuretic peptide elevated to $578 \mathrm{pg} / \mathrm{mL}$ (normal, o to 100), and lactate dehydrogenase elevated to 1,326 IU/L (normal, 263 to 450). Prothrombin time (PT) was elevated slightly above normal, with normal partial thromboplastin time (PTT). A peripheral blood smear showed mild anisopoikilocytosis consisting of elliptocytes, suggesting hemolytic anemia. On chest computed 

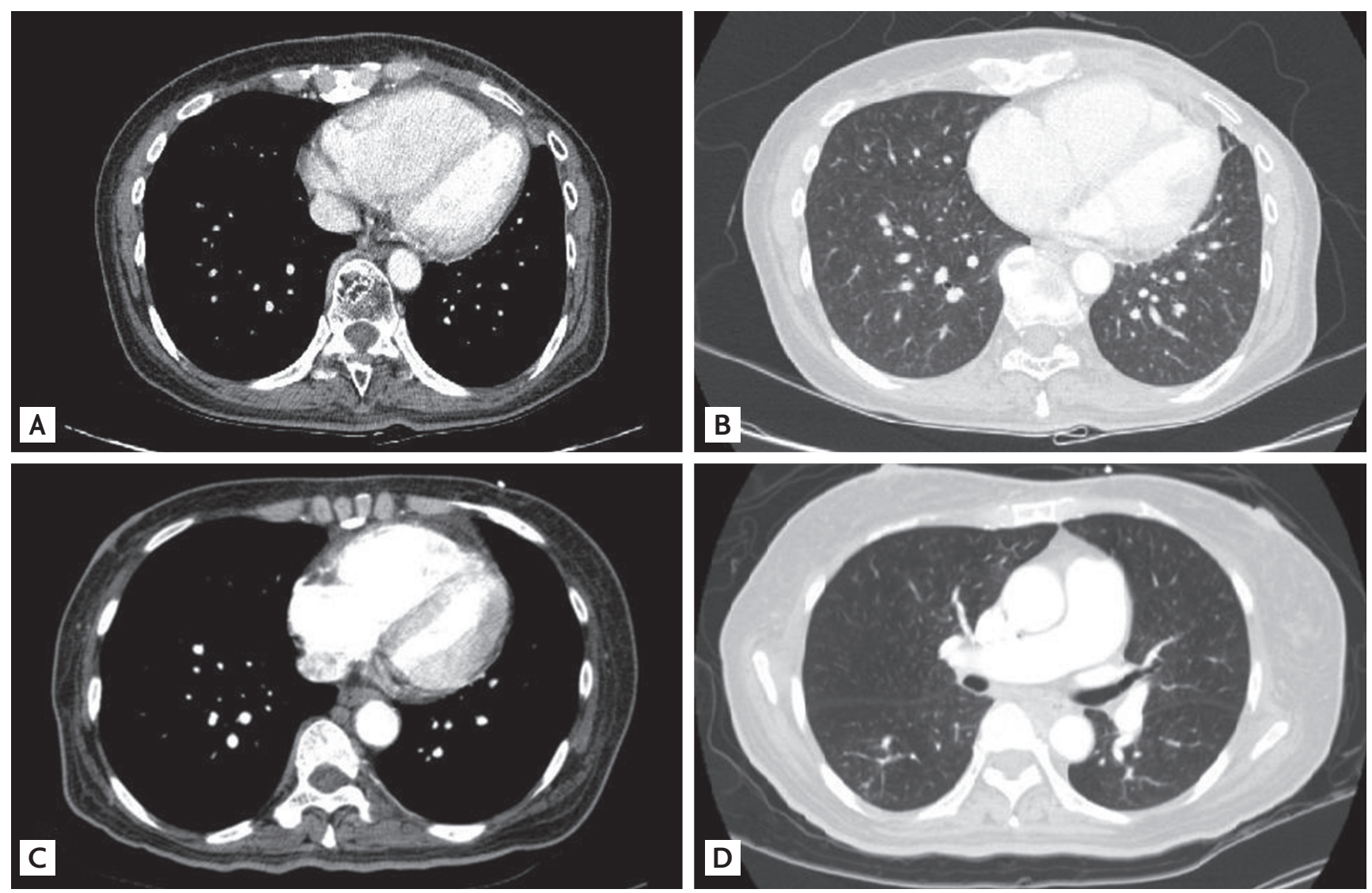

Figure 1. Computed tomography showed multiple centrilobular nodules with tree-in-bud appearances in cases 1, 2. A mediastinal setting showed a dilated right ventricle and a compressed D-shaped left ventricle in cases 1 and 2, respectively (A, C). A lung setting showed multiple ill-defined centrilobular nodules with tree-in-bud appearances in cases 1 and 2, respectively (B, D).

tomography (CT), there was new appearance of multiple, ill-defined centrilobular nodules in both lungs, but neither lymphangitic lung metastases nor pulmonary thromboembolism (PTE) were detected (Fig. 1A and 1B). A transthoracic echocardiogram (TTE) showed a D-shaped left ventricle with normal left ventricular systolic function with right ventricular enlargement, moderate tricuspid regurgitation, and moderate resting pulmonary hypertension with an estimated right ventricular systolic pressure of $63 \mathrm{mmHg}$, indicating right heart failure. PTTM was diagnosed based on right heart failure with pulmonary hypertension; the presence of underlying malignancy; no other significant causes of pulmonary hypertension; and PTE, primary lung disease, and valvular heart disease. Three days after admission, treatment was initiated with oxygen therapy and chemotherapy with vinorelbine $\left(25 \mathrm{mg} / \mathrm{m}^{2}\right.$ on days 1 and 8 , and carboplatin, area under the curve 5 , on day 1 , repeated every 3 weeks). Worsening dyspnea and hypoxia lead to admis- sion to the intensive care unit, 2 hours after chemotherapy injection. Despite chemotherapy administration, the patient died of right heart failure 4 days after admission.

The second case was a 58 -year-old woman who presented to the Emergency Department with altered mental status. She had a history of breast cancer with right breast conserving surgery performed at another hospital 9 years earlier. One year before admission, she was diagnosed with recurrent cancer in the remaining breast tissue; ultrasonography revealed multiple right axillary lymph node metastases. However, the patient refused further work-up and treatment. On this admission, she presented with stage IV metastatic breast cancer, including bilateral supraclavicular, cervical, and paraaortic lymph node and multiple bone metastases. Blood examination confirmed hyperglycemia, ketonuria, and metabolic acidosis. Therefore, the initial diagnosis was metabolic encephalopathy caused by infection, dehydration, and diabetic ketoacidosis (DKA). Brain magnet- 


\section{KJIM}

The Korean Journal of Internal Medicine Vol. 32, No. 1, January 2017
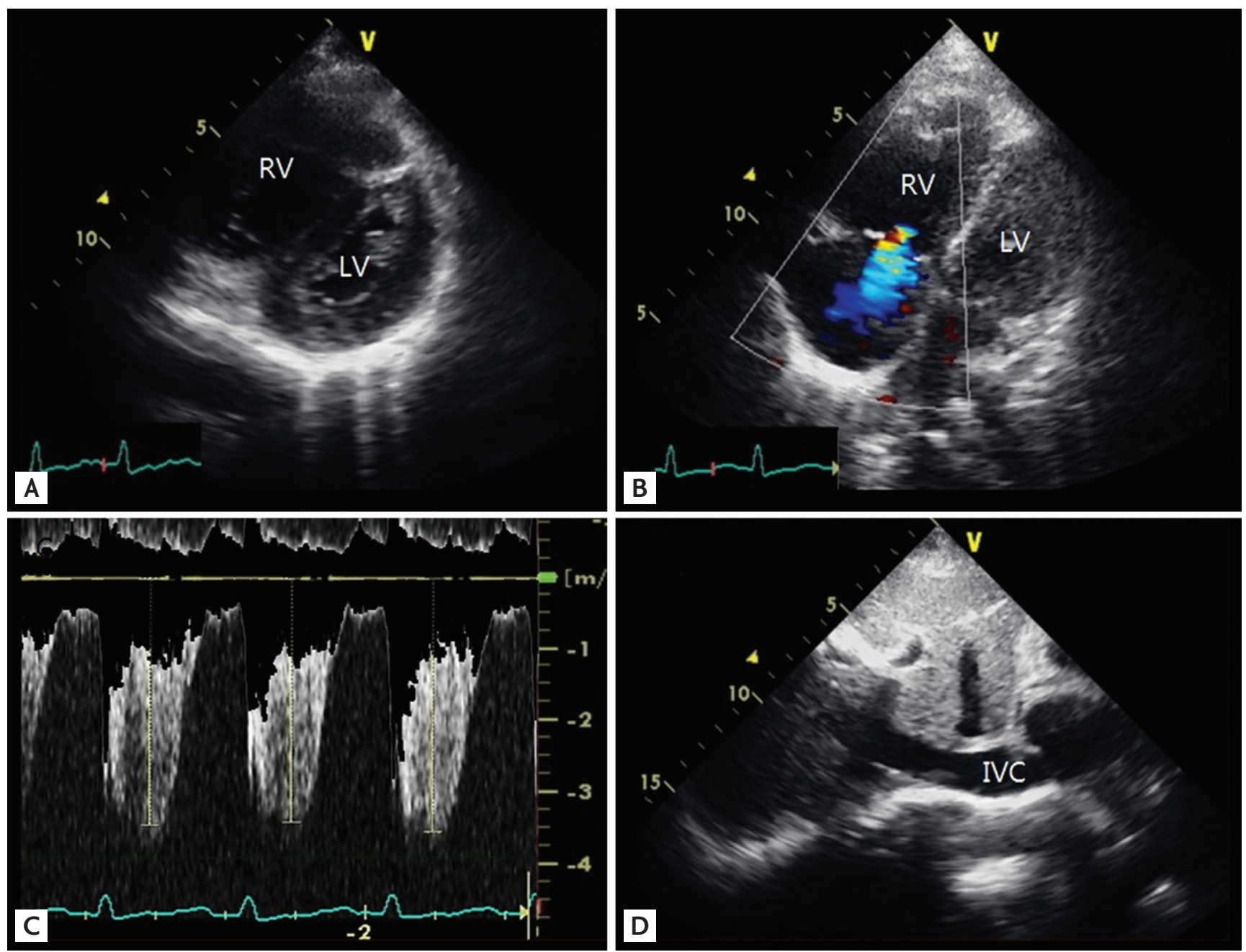

Figure 2. Echocardiography in case 2. (A) Parasternal short axis view, mid-ventricular plane on transthoracic echocardiography reveals a D-shaped LV throughout the systolic and diastolic period. (B) The 2-dimensional and color Doppler comparative focused image of the apical 4-chamber view showed mild-to-moderate tricuspid regurgitation. (C) The peak tricuspid regurgitation velocity was $3.7 \mathrm{~m} / \mathrm{sec}$, pressure gradient $55 \mathrm{mmHg}$, indicating pulmonary hypertension. (D) The dilated inferior vena cava (IVC) diameter in case 1 was $21 \mathrm{~mm}$ without inspiratory collapse. RV, right ventricle; LV, left ventricle.

ic resonance imaging revealed a cerebral venous sinus thrombus, possibly related to the hypercoagulable status of her systemic circulation. Although DKA was corrected, mental status deterioration continued. Furthermore, she rapidly developed progressive dyspnea and hypoxia. Pulmonary embolism chest CT showed no PTE, deep vein thrombosis, or other significant cause for dyspnea; however, nonspecific multiple centrilobular nodules were evident (Fig. 1 C and $1 \mathrm{D}$ ). TTE showed D-shaped left ventricle with normal left ventricular systolic function with right ventricular enlargement, moderate tricuspid regurgitation, and severe resting pulmonary hypertension with estimated right ventricular systolic pressure of $80 \mathrm{mmHg}$, indicating acute cor pulmonale (Fig. 2). Blood examination showed D dimer elevation to $>20 \mu \mathrm{g} / \mathrm{mL}$, with elevated brain natriuretic peptide level of $452 \mathrm{pg} /$ $\mathrm{mL}$. Fibrin degradation product was elevated to $120 \mu \mathrm{g} /$ $\mathrm{mL}$ (normal, o to 5), and the fibrinogen level was elevated to $623 \mathrm{mg} / \mathrm{dL}$ with a slightly increased PT of 16 seconds (international normalized ratio, 1.33) and normal PTT. These measures suggested disseminated intravascular coagulation (DIC). A peripheral blood smear revealed burr cells and schistocytes, suggesting microangiopathic hemolytic anemia (MAHA). Seven days after admission, the patient had desaturation and PTTM was considered. Despite anticoagulation and oxygen therapy, her respiration worsened and she died of right heart failure 8 days after admission. Despite a timely antemortem diagnosis 
and chemotherapy or anticoagulation treatment, the two patients died from acute cor pulmonale 4 and 8 days after admission, respectively.

PTT'M is a rare disease. From evaluation of an autopsy series, PTTM incidence varies from $2.4 \%$ to $26 \%$ in solid malignancies $[2,3]$. PTTM was caused by tumor cell infiltration into small pulmonary arterioles and by paraneoplastic thrombosis and fibrocellular intimal hyperplasia. The time of occurrence is variable, but it rarely presents as the initial manifestation of disease. The first clinical symptom is usually progressive dyspnea. The median patient survival time following oxygen treatment was 9 days [3]. With a proper antemortem diagnosis and chemotherapy, one patient with gastric cancer survived for 7 months after PTTM diagnosis; however, long-term survival is extremely rare [4]. PT'TM can simultaneously present with DIC and MAHA. Paraneoplastic thrombosis, DIC, and MAHA can be induced by undefined procoagulant factors released from cancer cells. Both patients showed features of DIC and MAHA. The second PT'TM case developed dural venous sinus thrombosis. Dural venous sinus thrombosis is associated with multiple risk factors, including systemic hypercoagulability. To the best of our knowledge, this is the first report of the co-occurrence of dural venous thrombosis and PTTM. Cerebral venous thrombosis and PTTM could develop because of paraneoplastic hypercoagulability. Most of the chest CT findings in patients with PTTM are nonspecific. There could be consolidation, ground-glass opacity, small nodules, and a tree-in-bud appearance [4]. The tree-in-bud centrilobular nodules were observed in both of our cases.

Differential PTTM diagnosis with lymphangitic lung metastases and PTE are crucial. Furthermore, $85.7 \%$ of PTTM cases occur coincidently with lymphangitic lung metastases [1]. However, isolated lymphangitic lung metastases without PTTM typically have an insidious and longer evolution; therefore, they rarely cause acute cor pulmonale. PTE differs from PTTM in terms of pulmonary vasculature location, involving larger pulmonary arteries and the absence of intimal hyperplasia. Although PTE may cause pulmonary hypertension and right heart failure, the clinical course of PTE is reversible and can be resolved with anticancer and heparin therapies. The clinical PTTM diagnosis may be possible with a high index of suspicion and exclusive diagnosis in patients who develop acute or subacute cor pulmonale. Definitive pathologic diagnosis through an invasive surgical procedure is not necessary for PTTM diagnosis because it is time consuming and the severity of the patient's condition typically excludes performing invasive diagnostic procedures.

Although PT'TM diagnosis is not difficult, treatment and reversal of right heart failure, the main cause of death, is challenging. Intimal hyperplasia and fibromuscular proliferation of small arterioles are nearly irreversible pathologies regardless of treatment [5]. Irreversible pulmonary vascular changes causing acute cor pulmonale result in very rapid clinical deterioration in these patients with no time for anticancer treatment. Therefore, very early diagnosis before the occurrence of irreversible pulmonary vascular changes is crucial. In a recent autopsy series, vascular endothelial growth factor (VEGF), tissue factor and platelet derived growth factor (PDGF) were overexpressed in $97 \%, 100 \%$, and $62 \%$ of cases, respectively [3]. PDGF blockade with STI571, a tyrosine kinase inhibitor, reversed pulmonary vascular changes and cor pulmonale in an experimental animal model [5]. Blocking the activity of cytokines such as VEGF and PDGF in addition to standard chemotherapy may help in effective PTTM treatment.

In summary, PTTM with acute cor pulmonale can be diagnosed with a high index of suspicion and exclusive diagnosis, including the presence of pulmonary hypertension, underlying malignancy, and the absence of other causes of pulmonary hypertension, such as chronic obstructive lung disease and valvular heart disease. Presence of MAHA and DIC were helpful for diagnosis, although a work-up for underlying malignancy should be warranted for acute or subacute cor pulmonale without specific lung or heart disease. However, very early diagnosis, before the development of irreversible pulmonary hypertension and vascular changes, and timely treatment are challenging [5].

Keywords: Breast neoplasms; Thrombi; Pulmonary heart disease

\section{Conflict of interest}

No potential conflict of interest relevant to this article was reported. 


\section{REFERENCES}

1. von Herbay A, Illes A, Waldherr R, Otto HF. Pulmonary tumor thrombotic microangiopathy with pulmonary hypertension. Cancer 1990;66:587-592.

2. Montero A, Vidaller A, Mitjavila F, Chivite D, Pujol R. Microscopic pulmonary tumoral embolism and subacute cor pulmonale as the first clinical signs of cancer. Acta Oncol 1999;38:1116-1118.
3. Uruga H, Fujii T, Kurosaki A, et al. Pulmonary tumor thrombotic microangiopathy: a clinical analysis of 30 autopsy cases. Intern Med 2013;52:1317-1323.

4. Miyano S, Izumi S, Takeda Y, et al. Pulmonary tumor thrombotic microangiopathy. J Clin Oncol 2007;25:597599.

5. Schermuly RT, Dony E, Ghofrani HA, et al. Reversal of experimental pulmonary hypertension by PDGF inhibition. J Clin Invest 2005;115:2811-2821. 